\title{
ADAPTAÇÃO CULTURAL DO CRITICAL NURSING SITUATION INDEX PARA A CULTURA BRASILEIRA*
}

\author{
Anderson Neri Guido' ${ }^{1}$ Edinêis de Brito Guirardello²
}

\begin{abstract}
RESUMO: Objetivo: traduzir e adaptar o Critical Nursing Situation Index para a cultura brasileira. Método: estudo metodológico que empregou as etapas de tradução, síntese das traduções, retro tradução, avaliação por comitê de juízes e pré-teste com a participação de 30 enfermeiros. Realizado no período de agosto de 2016 a abril de 2017 numa Unidade de Terapia Intensiva na cidade de Campinas-SP. Resultados: obteve-se a versão brasileira do instrumento. Avaliação das equivalências semântica, idiomática, cultural e conceitual pelo comitê resultou em uma taxa de concordância $\geq 80 \%$. Dos 84 itens que compõem o instrumento, quatro sofreram modificações. O tempo médio de preenchimento do instrumento na etapa de pré-teste foi de 16,7 minutos (Desvio-padrão $\pm 7,4$ ). Conclusão: $O$ processo metodológico de tradução e adaptação cultural do Critical Nursing Situation Index foi concluído com sucesso, e o instrumento apresentou grau satisfatório de compreensão global e aplicabilidade.
\end{abstract}

DESCRITORES: Cuidados críticos; Segurança do paciente; Enfermagem; Tradução; Indicadores de serviços.

\section{CULTURAL ADAPTATION OF THE CRITICAL NURSING SITUATION INDEX FOR THE BRAZILIAN CULTURE}

ABSTRACT: Objective: to translate and adapt the Critical Nursing Situation Index for the Brazilian culture. Method: methodological study that used the stages of translation, synthesis of translations, back-translation, evaluation by committee of judges and pre-test with the participation of 30 nurses. Performed in the period from August 2016 to April 2017 in an Intensive Care Unit in the city of Campinas-SP. Results: the Brazilian version of the instrument was obtained. Assessment of semantic, idiomatic, cultural and conceptual equivalence by the committee resulted in a concordance rate $\geq 80 \%$. Of the 84 items that comprise the instrument, four underwent modifications. The mean completion time of the instrument in the pre-test stage was 16.7 minutes (Standard deviation \pm 7.4 ). Conclusion: The methodological process of translation and cultural adaptation of the Critical Nursing Situation Index was completed successfully, and the instrument presented a satisfactory degree of global comprehension and applicability.

KEYWORDS: Intensive care; Patient safety; Nursing; Translation; Service indicators.

\section{EADAPTACIÓN CULTURAL DEL CRITICAL NURSING SITUATION INDEX A LA CULTURA BRASILEÑA}

RESUMEN: Objetivo: Traducir y adaptar el Critical Nursing Situation Index a la cultura brasileña. Método: Estudio metodológico aplicando las etapas de traducción, síntesis de las traducciones, retrotraducción, evaluación por comité de expertos y prueba piloto, con participación de 30 enfermeros. Realizado en el período de agosto de 2016 a abril de 2017 en una Unidad de Terapia Intensiva en la ciudad de CampinasSP. Resultados: Se obtuvo la versión brasileña del instrumento. La evaluación de equivalencias semántica, idiomática, cultural y conceptual por parte del comité arrojó una tasa de concordancia $\geq 80 \%$. De los 84 ítems que integran el instrumento, 4 sufrieron modificaciones. El tiempo promedio de completado del instrumento en la etapa de prueba piloto fue de 16,7 minutos (Desvío Estándar \pm 7,4). Conclusión: El proceso metodológico de traducción y adaptación cultural del Critical Nursing Situation Index concluyó con éxito, y el instrumento obtuvo grado satisfactorio de comprensión global y aplicabilidad.

DESCRIPTORES: Cuidados Críticos; Seguridad del Paciente; Enfermería; Traducción; Indicadores de Servicios.

\footnotetext{
*Manuscrito extraído da dissertação de mestrado intitulada: Adaptação Cultural e Validação do Critical Nursing Situation Index para a Cultura Brasileira. Universidade Estadual de Campinas - UNICAMP, 2017.

Enfermeiro, Mestre em Enfermagem pela Faculdade de Enfermagem. Gerente de centro cirúrgico do Hospital Municipal Dr. Mário Gatti. Campinas-SP-Brasil.

${ }^{2}$ Enfermeira, Doutora em Enfermagem. Docente Associada da Faculdade de Enfermagem, Universidade Estadual de Campinas. Campinas-SP-Brasil.
}

Autor Correspondente:

Anderson Neri Guido

Universidade Estadual de Campinas, Faculdade de Enfermagem

R. Tessália Vieira de Camargo, 126. Cidade Universitária - Campinas, SP - Brasil - CEP - 13083887

E-mail: anderson_neri@hotmail.com
Recebido: 06/09/2017

Finalizado: 08/02/2017 


\section{INTRODUÇÃO}

A enfermagem possui importante papel na promoção da segurança do paciente ${ }^{(1)}$. Essa responsabilidade se intensifica no ambiente da Unidade de Terapia Intensiva (UTI) à medida que os profissionais que ali atuam precisam prestar atenção tanto na gravidade do paciente quanto na complexidade e variedade dos inúmeros equipamentos inerentes a esse ambiente ${ }^{(2)}$.

Devido a essas características, os serviços de terapia intensiva apoiam-se em uma série de protocolos que norteiam as atividades de tratamento e cuidado $^{(3)}$. O uso desses recursos favorece a qualidade do cuidado, reduz o tempo de internação e, como consequência, os custos do serviço ${ }^{(4)}$. Por protocolo, entendese uma estrutura clara ou abordagem algorítmica do cuidado que proporciona aos membros da equipe multiprofissional instruções precisas a serem seguidas, constituindo ações individuais ou agrupadas em pacotes (bundles) de modo a organizar o cuidado ou estabelecer um caminho clínico ${ }^{(5)}$.

O uso de protocolos pode reduzir significativamente os riscos da assistência, como demonstrado por Pronovost (2006) e seus colaboradores, que, depois de instaurar um bundle de cinco cuidados baseados em evidências clínicas como lavagem de mãos, precaução de barreira máxima para inserção do cateter central, limpeza da pele com solução de clorexidina, evitar o sítio femoral se possível e remoção imediata de cateteres desnecessários, recomendados pelo Center of Diseas e Control (CDC), reduziram a taxa de incidência de infecção de corrente sanguínea de 0,62 para 0,34 em 18 meses $^{(6)}$.

Um estudo norte-americano estima que anualmente ocorram 82 mil infecções de corrente sanguínea associadas ao cateter central, sendo que 31.665 mortes são atribuídas a esse tipo de evento adverso. Tal situação gera um custo médio anual por caso que vai de US\$11.971 a US\$56.000 para atender pacientes que desenvolvem esse tipo de infecção ${ }^{(7)}$, de modo que utilizar esse bundle, além de proporcionar assistência de melhor qualidade, permite a economia de recursos ao evitar condição tão prejudicial ao paciente.

Outro estudo aponta que os erros relacionados à assistência à saúde podem ocupar a terceira maior causa de morte nos Estados Unidos ${ }^{(8)}$. Portanto, para garantir assistência segura ao paciente é necessário mensurar a performance da equipe de saúde, por meio de métodos válidos e confiáveis, capazes de aferir a qualidade do cuidado prestado ${ }^{(9)}$.

Durante a execução de suas atividades diárias, os profissionais de enfermagem realizam constantes visitas à beira do leito do paciente, e, por ocupar essa posição privilegiada na atenção à saúde, esse profissional tem a capacidade de identificar e corrigir diversas situações consideradas críticas no cuidado de enfermagem ${ }^{(10)}$. Tais situações são definidas como qualquer evento observado, que não corresponde ou está em desacordo com protocolos e padrões de enfermagem considerados como sendo boas práticas clínicas, adotados no âmbito da execução de atividades de terapia intensiva ${ }^{(11)}$.

O Critical Nursing Situation Index (CNSI) é um instrumento que tema finalidade de mensurar situações críticas de enfermagem ocorridas durante a assistência ao paciente adulto ${ }^{(11)}$. Desenvolvido para uso em UTI adulto e posteriormente adaptado para UTI pediátrica ${ }^{(12)}$, esse instrumento é considerado um dos pilares da iniciativa segurança em primeiro lugar do Erasmus MC - Sophia Children's Hospital, por proporcionar a avaliação e atualização dos protocolos de terapia intensiva, além de julgar o impacto da adesão ou não adesão a esses protocolos na incidência de eventos adversos relacionados à assistência à saúde ${ }^{(13)}$.

O instrumento é composto de 84 descrições de situações críticas de enfermagem, divididas em oito domínios: cuidados básicos de enfermagem em UTI (14 itens), ventilação mecânica (20 itens), acessos venosos (infusão e medidas) (10 itens), administração de fluidos (cinco itens), ritmo cardíaco e circulação (oito itens), medicação (10 itens), nutrição enteral (seis itens), cuidados de higiene e controle de dispositivos $(11 \text { itens })^{(11)}$.

As opções de respostas para cada item observado são: verdadeiro (presença de situação crítica), falso (ausência de situação crítica) ou não aplicável. A soma dos itens classificados como verdadeiros refletem o número de situações críticas, enquanto a soma dos itens identificados como verdadeiros e falsos determinam o número de itens em risco. 
O instrumento gera um índice, que é a porcentagem dos itens verdadeiros em relação aos itens em risco. Quanto maior o índice, mais desvios de protocolos foram encontrados, consequentemente, pior é a qualidade da assistência prestada ${ }^{(11)}$.

Com o propósito de disponibilizar uma ferramenta capaz de avaliar a adesão aos protocolos institucionais e instrumentalizar as equipes de enfermagem de terapia intensiva para executar tais avaliações, o presente estudo teve como objetivo realizar a tradução e a adaptação cultural do Critical Nursing Situation Index (CNSI) para a cultura brasileira.

\section{MÉTODO}

Trata-se de um estudo metodológico de tradução e adaptação cultural do CNSI para a realidade brasileira. Para o processo de tradução e adaptação cultural, seguiram-se os passos de tradução, síntese das traduções, retro tradução, avaliação por comitê de juízes e pré-teste ${ }^{(14)}$.

Na primeira etapa do processo de adaptação cultural, ocorreu a tradução do instrumento por dois tradutores independentes, ambos com fluência na língua inglesa e a língua portuguesa do Brasil como língua materna. Ressalta-se que um dos tradutores não recebeu informações sobre os objetivos e conceitos que envolvem o instrumento. Esta etapa resultou em duas versões (T1 e T2). Em seguida, realizou-se a síntese das versões pelos pesquisadores, obtendo-se a versão-síntese (T12).

A retro tradução foi realizada por dois tradutores independentes, com fluência na língua portuguesa do Brasil e a língua inglesa como materna. Eles tiveram como objetivo traduzir a versão-síntese de volta para o inglês. Esse procedimento tem como finalidade checar a validade da tradução e verificar a existência de discrepâncias no significado e no conteúdo entre o instrumento original e o instrumento traduzido ${ }^{(14)}$.

As versões produzidas foram submetidas à avaliação por um comitê de seis juízes, composto de um metodologista, um lingüista, dois enfermeiros atuantes em terapia intensiva e dois fisioterapeutas especialistas em terapia intensiva. O objetivo desta etapa foi avaliar as equivalências semântica, idiomática, conceitual e cultural entre versão original e versão-síntese, obtendo-se, também, a validação do conteúdo do instrumento.

A equivalência semântica visa à transferência de significados entre as línguas, considerando a gramática e o vocabulário, de modo a atingir efeito similar nos sujeitos que utilizam diferentes línguas. A equivalência idiomática é um desdobramento da equivalência semântica e considera as adaptações necessárias para traduzir expressões que não apresentam significado em seu sentido literal, são expressões coloquiais, informais ou gírias, que muitas vezes são difíceis ou inviáveis de traduzir literalmente. As equivalências conceitual e cultural objetivam explorar os conceitos principais e suas definições nas populações envolvidas ${ }^{(15)}$.

Os juízes receberam um convite formal com orientações sobre o trabalho a executar, assim como um quadro contendo todas as versões produzidas nas etapas anteriores. Foram solicitados a avaliar os 84 itens do instrumento de forma quantitativa ao julgarem as equivalências em uma escala dividida em quatro graus de concordância (1 - item não equivalente, 2 - impossível avaliar sem revisão do item, 3 - equivalente, mas necessita alterações, 4 - absolutamente equivalente). Foi estabelecido um grau de consenso $\geq 80 \%{ }^{(16)}$ para cada item avaliado.

Os itens que a princípio não atingiram essa taxa de concordância foram, então, discutidos em reunião com os juízes, revistos e modificados, até a obtenção de consenso. Além disso, os juízes julgaram o instrumento de forma qualitativa, definindo, assim, a sua validade de conteúdo. Ao final desta etapa, gerou-se a versão pré-final do instrumento.

Na etapa de pré-teste participaram 30 enfermeiros da UTI de um hospital universitário do interior do estado de São Paulo, que receberam instruções específicas para a aplicação da versão pré-final do instrumento. Foram solicitados a avaliar o tempo de preenchimento e a aplicabilidade do instrumento. Além disso, foram solicitados a expressar suas opiniões sobre itens que necessitavam de revisão. 
Este estudo obteve a autorização do autor do instrumento para realizar o procedimento de tradução e adaptação cultural, bem como a aprovação do comitê de ética em pesquisa da Universidade Estadual de Campinas (UNICAMP), com parecer número 1.693.664.

\section{RESULTADOS}

As etapas iniciais de tradução, síntese e retro tradução foram realizadas sem dificuldades, com apenas quatro itens que obtiveram percentual de concordância abaixo de 80\%. Os itens 17 e 63 apresentaram 50\% de concordância em todas as equivalências avaliadas, enquanto o item 4 apresentou concordância de $67 \%$ nas equivalências semântico-idiomática; e o título do oitavo domínio (T8) obteve $67 \%$ de concordância na categoria equivalência cultural (Quadro 1).

Quadro 1 - Modificação dos itens do CNSI após reunião presencial dos juízes. Campinas, SP, Brasil, 2017

\begin{tabular}{|c|c|c|c|}
\hline Itens & $\begin{array}{c}\text { Critical Nursing Situation } \\
\text { Index }\end{array}$ & Versão-síntese & Versão final \\
\hline 4 & $\begin{array}{l}\text { Entrance of the isolation } \\
\text { room is not marked as such }\end{array}$ & $\begin{array}{l}\text { O quarto em precaução não possui } \\
\text { sinalização conforme protocolo da } \\
\text { instituição }\end{array}$ & $\begin{array}{l}\text { O quarto em precaução (isolamento) } \\
\text { não está identificado como tal }\end{array}$ \\
\hline 17 & $\begin{array}{l}\text { No manual inflation } \\
\text { according to protocol }\end{array}$ & $\begin{array}{l}\text { Sem fisioterapia respiratória de } \\
\text { acordo com o protocolo }\end{array}$ & $\begin{array}{c}\text { Ausência de insuflação manual } \\
\text { (manobra com } \mathrm{AMBU}^{\circledR} \text { ) de acordo com } \\
\text { o protocolo }\end{array}$ \\
\hline 63 & $\begin{array}{l}\text { No supported continuous } \\
\text { flush infusion in patient } \\
\text { with cardiogenic } \\
\text { medication } \\
\end{array}$ & $\begin{array}{l}\text { Sem infusão contínua para lavagem } \\
\text { de suporte em pacientes com } \\
\text { medicação cardiogênica }\end{array}$ & $\begin{array}{c}\text { Ausência de infusão contínua para } \\
\text { lavagem de suporte em pacientes } \\
\text { recebendo droga(s) vasoativa(s) }\end{array}$ \\
\hline T8 & $\begin{array}{l}\text { Hygienic care and control } \\
\text { of parts and devices items }\end{array}$ & $\begin{array}{l}\text { Cuidados de higiene e controle de } \\
\text { partes e dispositivos }\end{array}$ & $\begin{array}{c}\text { Cuidados de Higiene e Controle de } \\
\text { Dispositivos }\end{array}$ \\
\hline
\end{tabular}

Os itens apresentados no Quadro 1 foram modificados após reunião presencial com os membros do comitê de juízes e pesquisadores, possibilitando que esses os avaliassem qualitativamente.

Com relação ao item 4, as questões levantadas foram acerca das palavras precaução e isolamento, uma vez que ambas as palavras são utilizadas em diferentes realidades do território nacional. Foi então sugerido adicionar a palavra "isolamento" entre parênteses para facilitar a compreensão do item.

O item 17 apresentou dificuldade semelhante, pois no Brasil é muito comum o uso da marca registrada $\mathrm{AMBU}^{\circledR}$ para descrever o dispositivo de bolsa - válvula unidirecional - máscara utilizado para realizar procedimentos que necessitam de insuflação manual. Desse modo, optou-se pelo parêntese explicativo, mantendo a marca registrada desse tipo de equipamento.

Como o item 63 apresentou dificuldades por se tratar de uma prática incomum no Brasil, foi necessário recorrer ao autor da versão original do instrumento, de modo a adquirir maiores informações sobre esse protocolo, para obtenção da versão pré-final do processo de tradução e adaptação cultural.

Por fim, os juízes observaram que o uso da palavra partes, no título do oitavo domínio, não correspondia ao uso comum na cultura brasileira, optando por suprimir essa palavra na tradução. Depois desses ajustes, os itens revisados atingiram $100 \%$ de consenso, e as modificações foram incorporadas à versão pré-final do instrumento, a qual foi submetida ao pré-teste.

Durante o pré-teste, alguns enfermeiros relataram a dificuldade em reconhecer que a avaliação dos itens que compõem o domínio de ventilação mecânica era de sua competência. $O$ tempo de preenchimento médio do instrumento foi de 16,7 minutos $(\mathrm{dp} \pm 7,4)$, com tempo mínimo de preenchimento de 5 e máximo de 35 minutos. 
A compreensão das instruções para aplicação do instrumento e a facilidade para entender e assinalar seus itens estão demonstrados na Tabela 1, e os itens que compõem a versão do instrumento estão apresentado no Quadro 2.

Tabela 1 - Resultados da avaliação de aplicabilidade do instrumento na etapa de pré-teste.Campinas, SP, Brasil, 2017

\begin{tabular}{|c|c|c|c|c|c|c|c|c|c|c|}
\hline \multirow[t]{2}{*}{ Questões } & \multicolumn{2}{|c|}{$\begin{array}{l}\text { Discordo } \\
\text { Totalmente }\end{array}$} & \multicolumn{2}{|c|}{$\begin{array}{c}\text { Discordo } \\
\text { Parcialmente }\end{array}$} & \multicolumn{2}{|c|}{$\begin{array}{l}\text { Não tenho } \\
\text { Opinião }\end{array}$} & \multicolumn{2}{|c|}{$\begin{array}{c}\text { Concordo } \\
\text { Parcialmente }\end{array}$} & \multicolumn{2}{|c|}{$\begin{array}{l}\text { Concordo } \\
\text { Totalmente }\end{array}$} \\
\hline & $n$ & $\%$ & $\mathrm{n}$ & $\%$ & $\mathbf{n}$ & $\%$ & $n$ & $\%$ & $n$ & $\%$ \\
\hline $\begin{array}{l}\text { Eu achei fácil entender as } \\
\text { instruções do instrumento }\end{array}$ & 0 & 0 & 4 & 13,3 & 1 & 3,4 & 12 & 40 & 13 & 43,4 \\
\hline $\begin{array}{l}\text { Eu achei fácil entender os itens do } \\
\text { instrumento }\end{array}$ & 0 & 0 & 2 & 6,7 & 0 & 0 & 21 & 70 & 7 & 23,3 \\
\hline $\begin{array}{l}\text { Eu achei fácil assinalar os itens do } \\
\text { instrumento }\end{array}$ & 0 & 0 & 4 & 13,3 & 1 & 3,3 & 8 & 26,7 & 17 & 56,7 \\
\hline
\end{tabular}

Quadro 2 - Critical Nursing Situation Index (Versão brasileira). Campinas, SP, Brasil, 2017. (continua)

\section{Cuidados Básicos de Enfermagem em UTI}

1 Ausência de histórico/registro de culturas bacterianas após transferência de outro hospital

2 Cultura bacteriana atrasada por mais de 2 horas (apesar de pedido por escrito)

3 Ausência de avaliação de risco para úlceras por pressão

4 O quarto em precaução (isolamento) não está identificado como tal

5 Os olhos do paciente estão visivelmente contaminados

6 Uso incorreto da Escala de Coma de Glasgow

7 O paciente não é mobilizado de acordo com as instruções

8 A posição do paciente não está de acordo com as instruções

9 Ausência de evacuação por mais de 3 dias e sem nenhuma intervenção no $4^{\circ}$ dia

10 Ausência de controle do débito urinário para avaliação do balanço hídrico

11 Ausência de registro do turno anterior

12 Ausência de registro sobre família ou parentes

13 Ausência de registro sobre a altura e peso do paciente nos formulários da UTI

14 Ausência de registro atualizado de temperatura (últimas 48 horas)

\section{Ventilação Mecânica}

15 Discrepância entre o registro e o ajuste real dos parâmetros de ventilação mecânica

16 Ausência de registro da PEEP intrínseca, de hora em hora, durante ventilação de pressão controlada

17 Ausência de insuflação manual (manobra com $\mathrm{AMBU}^{\circledR}$ ) de acordo com o protocolo

18 Ausência de aspiração endotraqueal de acordo com o protocolo

19 Ausência de registro claro de mudanças nos parâmetros de ventilação mecânica

20 Reposicionamento do tubo endotraqueal em desacordo com o protocolo

21 Ausência de amostra de gasometria arterial em até 1 hora após remoção do tubo endotraqueal

22 Terapia de inalação durante ventilação mecânica não está de acordo com as instruções

23 Cabeceira do leito do paciente não está de acordo com o protocolo

24 Condensado visível (água ou secreção) entre a conexão do circuito respiratório e o tubo endotraqueal

25 Condensado acumulado (água ou secreção) no circuito respiratório 
26 Condensado visível (água ou secreção) no circuito inspiratório da ventilação mecânica

27 O sistema de umidificação não funciona (está desligado)

28 Ausência de monitorização da saturação de oxi-hemoglobina periférica (oximetria de pulso) e capnografia do paciente em posição prona

29 Ausência de sistema fechado de aspiração endotraqueal em pacientes na posição prona

30 Ausência de umidificador conectado ao fluxômetro de oxigênio na configuração básica do painel da UTI (suporte no caso de mau funcionamento do ventilador)

31 Ausência de sistema de aspiração endotraqueal completo (e funcionando) na configuração básica do painel da UTI

32 Ausência de solução estéril de $\mathrm{NaCL}$ 0,9\% para flush endotraqueal na configuração básica do painel da UTI

33 Ajuste de fluxo incorreto durante a ventilação mecânica no modo assistido

34 O ajuste máximo de pressão da ventilação mecânica excede os limites prescritos (seguros)

\section{Acessos Endovenosos (infusão e medidas)}

35 Ausência de registro da inserção do cateter venoso central

36 Ausência de registro da inserção do cateter arterial

37 Cateter de Swan-Ganz in situ por mais de 4 dias

38 Cateter central in situ em desacordo com o protocolo (tempo de permanência ou condição)

39 Cateter arterial in situ em desacordo com o protocolo (tempo de permanência ou condição)

40 Uma ou mais tampas faltando no acesso arterial

41 Uma ou mais tampas faltando no cateter de Swan-Ganz

42 Uma ou mais tampas faltando no acesso venoso periférico

43 Bolsa de soro vazia no sistema de bolsa pressórica

44 Pressão insuficiente no sistema de bolsa pressórica

\section{Administração de Fluidos}

45 Ausência de avaliação de balanço hídrico de acordo com o protocolo

46 Bolsa de sangue sem número de registro está instalada no paciente

47 Bolsa de sangue não foi conferida e checada por um segundo profissional de enfermagem

48 Volume de fluidos utilizado para lavagem (flush) não foi mensurado (ou está incorreto) no formulário de balanço hídrico da UTI

49 Nem todas as infusões administradas no paciente estão registradas no formulário da UTI

\section{Ritmo Cardíaco e Circulação}

50 Ausência do ECG de rotina feito na admissão

51 Pressão arterial invasiva não conferida com esfigmomanômetro de acordo com o protocolo

52 Ausência de estudo hemodinâmico realizado em pacientes com cateter de Swan-Ganz

53 Monitoramento incorreto do ritmo cardíaco (frequência)

54 Alarme sonoro do ritmo cardíaco está permanentemente desligado

55 Alarme sonoro para curvas de pressão está permanentemente desligado

56 Limites de alarme do ritmo cardíaco e pressão arterial não estão ajustados adequadamente

57 Ponto de referência e dispositivo de pressão invasiva não está nivelado na altura correta

\section{Medicação}

58 Medicações prescritas não foram administradas ou não estão checadas

59 Medicação endovenosa prescrita para infusão prolongada não conectada

60 Discrepância entre a taxa de infusão de medicação endovenosa prescrita e real

61 Medicação endovenosa de infusão prolongada conectada ao paciente não está anotada no formulário da UTI

62 Medicação endovenosa preparada sem dupla verificação e checagem de acordo com o protocolo

63 Ausência de infusão contínua para lavagem de suporte em pacientes recebendo droga(s) vasoativa(s) 
64 Via de acesso não utilizada não está tampada

65 Medicação endovenosa conectada no lúmen errado

66 Medicação endovenosa de via exclusiva combinada com outra medicação

67 Medicação endovenosa contínua combinada com medicação endovenosa intermitente

\section{Nutrição Enteral}

68 Ausência de registro de introdução do cateter enteral

69 Ausência de mensuração do resíduo gástrico durante terapia de nutrição enteral

70 Ingestão de menos de 75\% da alimentação enteral prescrita sem razão específica

71 Cateter enteral pós-pilórico não lavado de acordo com as instruções

72 Troca do frasco de dieta enteral excede o tempo permitido

73 Paciente com cabeceira do leito horizontal enquanto recebe dieta enteral

\section{Cuidados de Higiene e Controle de Dispositivos}

74 Dispositivo de dreno de tórax em aspiração vazando ar

75 Selo d'água do dispositivo de dreno de tórax ausente ou insuficiente

76 Dispositivos de inalação não trocados de acordo com o protocolo

77 Sistema fechado de aspiração traqueal não trocado de acordo com o protocolo

78 Equipamento de ventilação mecânica não trocado de acordo com o protocolo

79 Sistema de infusão de nutrição parenteral não trocado de acordo com o protocolo

80 Curativo do cateter venoso central não trocado de acordo com o protocolo

81 Curativo do cateter arterial não trocado de acordo com o protocolo

82 Sistema de mensuração de pressão venosa central (PVC) ou pressão arterial invasiva não trocados de acordo com o protocolo

83 Equipo de soro não trocado de acordo com o protocolo

84 Curativo de inserção de cateteres periféricos não trocados de acordo com o protocolo

\section{DISCUSSÃO}

Os instrumentos de medidas desenvolvidos em outras línguas e outras culturas não podem ser simplesmente traduzidos devido a importantes diferenças culturais e linguísticas. Portanto, o processo de tradução e adaptação cultural requer seguir as etapas recomendadas do modelo adotado(14), atentando para aspectos linguísticos, semânticos, culturais e conceituais ${ }^{(15)}$.

Neste estudo, as etapas de tradução, síntese, retro tradução, avaliação por comitê de juízes e pré-teste foram realizadas de forma satisfatória. Durante a reunião presencial, a composição multidisciplinar do comitê de juízes permitiu uma rica discussão dos itens e de sua tradução. A avaliação de equivalências foi realizada tanto de forma quantitativa, ao se estabelecer a concordância, quanto qualitativa, pois todos os juízes opinaram sobre a tradução dos itens do instrumento, fornecendo valiosas sugestões para a composição da versão pré-final.

Apenas o item 63, "Ausência de infusão contínua para lavagem de suporte em pacientes recebendo droga(s) vasoativa(s)", precisou ser abordado com o autor correspondente do instrumento original, além de ter demandado maior discussão durante a reunião presencial com os juízes. Esse item refere-se a uma técnica utilizada principalmente com sistemas de bomba de infusão de seringa, a qual é acoplada a uma bomba de infusão volumétrica, esta contendo solução salina que atua como veículo das medicações vasoativas altamente concentradas contidas nas seringas. Entretanto, essa prática não é comum em nossa cultura, e esse item acarretou diversos apontamentos, por parte dos enfermeiros participantes do pré-teste, sobre qual o significado de tal prática. 
É fundamental destacar que o CNSI pode e deve ser modificado segundo a realidade da instituição onde ele será aplicado. Tais adaptações são necessárias, uma vez que protocolos institucionais possuem particularidades e variam entre instituições de um mesmo país. Esses ajustes não comprometem a validade da ferramenta, sendo recomendados pelo autor do instrumento, já que o princípio do CNSI é baseado na mensuração de desvios ou erros observáveis e evitáveis no cuidado de enfermagem, não se tratando de uma lista imutável de itens que deve ser rigorosamente copiada para ser utilizada em outras instituições de saúde ${ }^{(16)}$.

A respeito da aplicabilidade do instrumento, a maioria dos enfermeiros concordou parcialmente ou totalmente que foi fácil entender as instruções e responder aos itens do instrumento. O tempo médio de 16,7 minutos para preenchimento do instrumento pode ser explicado pelo fato de que os enfermeiros preencheram o instrumento enquanto desempenhavam suas atividades durante o horário de trabalho e, portanto, tiveram que lidar com as costumeiras interrupções e demandas que surgiram ao longo do plantão.

Com relação ao domínio "ventilação mecânica", foi apontado por alguns enfermeiros que tais avaliações devem ser realizadas pela equipe de fisioterapia, pois muitos afirmaram "não conhecer" ou "não dominar" as informações ou regulagens de parâmetros utilizados no ventilador mecânico. Tal particularidade pode ser explicada devido ao fato de que o ambiente complexo da UTI precisa de educação continuada de qualidade para a equipe de enfermagem, inclusive envolvendo o uso de equipamentos como o ventilador ${ }^{(17)}$.

Um estudo realizado no Brasil apontou que as principais dificuldades relatadas pela equipe de enfermagem ao prestar cuidados ao paciente crítico em ventilação mecânica foram falta de conhecimento sobre os cuidados, falta de segurança ao manipular o ventilador e falta de cursos de aperfeiçoamento ${ }^{(18)}$.

Destaca-se que o CNSI, além de preencher a lacuna de instrumentos que possibilitam ao enfermeiro a quantificação da adesão ou não da equipe aos protocolos institucionais na cultura brasileira, favorece a prestação de cuidado seguro ao paciente.

Ressalta-se a necessidade de testes adicionais para avaliar as propriedades psicométricas, por meio da utilização de outras medidas de avaliação da confiabilidade e validade, considerando que o presente assegurou apenas a validade de conteúdo da ferramenta.

\section{CONCLUSÃO}

Este estudo realizou a adaptação cultural do CNSI para a cultura brasileira de maneira satisfatória. Tratase de uma ferramenta capaz de avaliar e quantificar a adesão da equipe de enfermagem de terapia intensiva aos protocolos institucionais, de modo identificar falhas que possam pôr em risco a segurança dos pacientes atendidos nessas unidades.

O processo de adaptação cultural seguiu a estrutura metodológica recomendada pela literatura, atingindo o objetivo proposto de elaborar a versão brasileira do instrumento.

Ressalta-se a necessidade de testes adicionais para avaliar as propriedades psicométricas, por meio da utilização de outras medidas de avaliação da confiabilidade e validade, considerando que o presente assegurou apenas a validade de conteúdo da ferramenta.

\section{REFERÊNCIAS}

1. Te Beest H, van der Starre C, Tibboel D, van Dijk M. Nursing protocol violations: detect, correct and communicate. Nurs Crit Care. [Internet] 2013;18(2) [13 de ago 2015]. Disponível: http://dx.doi.org/10.1111/j.1478-5153.2012.00533.x.

2. Zeraati M, Alavi NM. Designing and validity evaluation of Quality of Nursing Care Scale in Intensive Care Units. J Nurs Meas. [Internet] 2014;22(3) [acesso em 13 ago 2015]. Disponível: https://doi.org/10.1891/1061-3749.22.3.461. 
3. de Vos M, Graafmans W, Keesman E, Westert G, van der Voort PHJ. Quality measurement at intensive care units: which indicators should we use? J Crit Care. [Internet] 2007;22(4) [acesso em 13 ago 2015]. Disponível: http://dx.doi. org/10.1016/j.jcrc.2007.01.002.

4. Hammond JJ. Protocols and guidelines in critical care: development and implementation. Curr Opin Crit Care. [Internet] 2001;7(6) [acesso em 13 ago 2015]. Disponível: https://doi.org/10.1097/00075198-200112000-00017.

5. LeBlanc JM, Kane-Gill SL, Pohlman AS, Herr DL. Multiprofessional survey of protocol use in the intensive care unit. J Crit Care. [Internet] 2012;27(6) [acesso em 13 ago 2015]. Disponível: https://doi.org/10.1016/j.jcrc.2012.07.012.

6. Pronovost P, Needham D, Berenholtz S, Sinopoli D, Chu H, Cosgrove S, et al. An intervention to decrease catheter -related bloodstream infections in the ICU. N Engl J Med. [Internet] 2006;355(26) [acesso em 13 ago 2015]. Disponível: https://doi.org/10.1056/NEJMoa061115.

7. Pronovost PJ, Watson SR, Goeschel CA, Hyzy RC, Berenholtz SM. Sustaining Reductions in Central Line-Associated Bloodstream Infections in Michigan Intensive Care Units A 10-Year Analysis. Am J Med Qual. [Internet] 2015;31(3) [acesso em 13 ago 2015]. Disponível: http://doi.org/10.1177/1062860614568647.

8. Makary MA, Daniel M. Medical error-the third leading cause of death in the US. BMJ. [Internet] 2016;353 [acesso em 19 out 2017]. Disponível: https://doi.org/10.1136/bmj.i2139.

9. Pronovost PJ, Berenholtz SM, Ngo K, McDowell M, Holzmueller C, Haraden C, et al. Developing and pilot testing quality indicators in the intensive care unit. J Crit Care. [Internet] 2003;18(3) [acesso em 13 ago 2015]. Disponível: https://doi.org/10.1016/j.jcrc.2003.08.003.

10. Wassenaar A, Boogaard M, Hooft T, Pickkers P, Schoonhoven L. 'Providing good and comfortable care by building a bond of trust': nurses views regarding their role in patients' perception of safety in the Intensive Care Unit. J Clin Nurs. [Internet] 2015;24(21-22) [acesso em 13 ago 2015]. Disponível: https://doi.org/10.1111/jocn.12995.

11. Binnekade JM, de Mol BA, Kesecioglu J, de Haan RJ. The Critical Nursing Situation Index for safety assessment in intensive care. Intensive Care Med. [Internet] 2001;27(6) [acesso em 13 ago 2015] Disponível: https://doi.org/10.1007/ s001340100947.

12. de Neef M, Bos AP, Tol D. Safety as a criterion for quality: The Critical Nursing Situation Index in paediatric critical care, an observational study. Intensive Crit Care Nurs. [Internet] 2009;25(6) [acesso em 13 ago 2015]. Disponível: https://doi.org/10.1016/j.iccn.2009.08.002.

13. Van der Starre C, Van der Tuijn Y, Tibboel D. Patient safety management system in pediatric ICUs. Springer. [Internet] 2006 [acesso em 13 ago 2015] Disponível: https://doi.org/10.1007/3-540-33396-7_70.

14. Beaton D, Bombardier C, Guillemin F, Ferraz MB. Recommendations for the cross-cultural adaptation of the DASH \& QuickDASH outcome measures. Institute for Work \& Health. [Internet] 2007;1(1) [acesso em 13 ago 2015] Disponível: http://www.dash.iwh.on.ca/sites/dash/files/downloads/cross_cultural_adaptation_2007.pdf

15. Epstein J, Santo RM, Guillemin F. A review of guidelines for cross-cultural adaptation of questionnaires could not bring out a consensus. J Clin Epidemio. [Internet] 2015;68(4) [acesso em 20 ago 2016] Disponível: https://doi.org/10.1016/j.jclinepi.2014.11.021.

16. Binnekade JM, Vroom MB, de Mol BA, De Haan RJ. The quality of intensive care nursing before, during, and after the introduction of nurses without ICU-training. Heart Lung. [Internet] 2003;32(3) [acesso em 13 ago 2015]. Disponível: https://doi.org/10.1016/S0147-9563(03)00032-3.

17. Guilhermino MC, Care GCC, Inder KJ, Epid GDC, Sundin D, Kuzmiuk L, et al. Nurses' perceptions of education on invasive mechanical ventilation. J Contin Educ Nurs. [Internet] 2014;45(5) [acesso em 20 jun 2016]. Disponível: https:// doi.org/10.3928/00220124-20140417-01.

18. Melo EM, Teixeira CS, Oliveira RT, Almeida DT, Veras Jeglf FNM. Cuidados de enfermagem ao utente sob ventilação mecânica internado em unidade de terapia intensiva. Rev Enf Ref. [Internet] 2014;4(1) [acesso em 13 ago 2015]. Disponível: http://www.scielo.mec.pt/scielo.php?script=sci_abstract\&pid=S0874-02832014000100007\&lng=pt\&nrm=i http://dx.doi.org/10.12707/RIII1316. 